\title{
DIRECT NUMERICAL SIMULATION OF PARTICLE SEDIMENTATION IN TWO-PHASE FLOW UNDER THERMAL CONVECTION
}

\author{
J. Z. CHANG, H. T. LIU, T. X. SU and M. B. LIU \\ School of Mechatronice Engineering \\ North University of China, Taiyuan 030051, China \\ changjz01@nuc.edu.cn \\ M. B. LIU* \\ Key Laboratory for Hydrodynamics and Ocean Engineering \\ Institute of Mechanics \\ Chinese Academy of Sciences \\ Beijing 100190, China \\ The State Key Laboratory for Nonlinear Mechanics \\ Institute of Mechanics \\ Chinese Academy of Sciences \\ Beijing 100190, China \\ liumoubin@imech.ac.cn
}

Received 16 November 2009

Accepted 16 August 2010

\begin{abstract}
This paper presents a direct numerical simulation of particle sedimentation in two-phase flow with thermal convection. The sedimentation processes of elliptical particles are investigated in three different scenarios with isotherm, hot, and cold Newtonian fluids. We demonstrate that different particle shapes and orientations can result in quite different flow behaviors. Some interesting results have been obtained, which are very helpful for better understanding of the particle sedimentation processes.
\end{abstract}

Keywords: Direct numerical simulation; two-phase flows; thermal convection; particle sedimentation.

\section{Introduction}

Multiphase flows of particle sedimentation with thermal convection widely exist in industrial and natural processes and are fundamentally important both for academic researches and engineering applications [Jog et al. (1996); Lin et al. (2002); Liu et al. (2004)]. They are associated with complex flow physics including reaction, heat and mass transfers, thermal and compositional convections, moving and deformable

${ }^{*}$ Corresponding author. 
interfaces, and particle-particle interactions and, therefore, they are difficult for numerical simulations [Codina and Houzeaux (2009); Anthony (2009); Takeuchi et al. (2008)].

Current knowledge of flow and transport in reaction and multiphase system is largely empirical and theoretical modeling remains phenomenological [Mezhericher et al. (2008); Deen and Annaland (2007)]. In addition, most of the current studies concentrate on circular particles. However, practical applications involve more noncircular particles and forces on particles are usually revised by "equivalent diameter" and degree of sphericity [Jae and Ronald (2004); You et al. (2003)]. Traditional ways to solve particle sedimentation problems rely on theoretical analysis and laboratory findings [Mcleod et al. (1996); Kuehn and Goldstein (1976)] and are limited to simple cases. Computer simulations have been playing an important role in investigating more complicated cases with dispersed two-phase flows using numerical models such as homogeneous multi-phase model, mean-shift model, and two-fluid model. These numerical models cannot describe the inner structure and details of the multiphase flow field, and are not able to investigate the particle-fluid inter-coupling mechanism accurately due to the over-simplified hypothesis such as stationery and single particle without considering thermal convection effects [Acrivos and Taylor (1962); Shigeo and Hideo (2001)]. It is necessary to establish numerical models that can investigate the coupled physics with fluid flow, thermal convection, and mass transfer of moving particles [Gan et al. (2003); Feng et al. (1994)].

In this paper, the sedimentation processes of elliptical particles have been investigated by using a direct numerical simulation method and some revealing results have been obtained.

\section{Problem Description and Numerical Methods}

A circular particle of diameter $2 d$ or an elliptical particle with a major axis $2 d$ and a minor axis $d$ is released from the central line of a channel with zero initial velocity. The size of the channel is $L$ and $8 d$ in $x$ and $y$ directions (Fig. 1) and the channel is filled with a Newtonian fluid. The distances from the initial particle position to the channel top/bottom are $15 d$. The initial fluid temperature is $T_{0}$ and the solid particle temperature is fixed at $T_{s}$. The particle is incorporated into a hot or cool fluid of the same material, $G r=1,143, \operatorname{Pr}=0.7$. The particle destiny $\rho_{s}$ is greater than the fluid destiny $\rho_{o}$ and the particle starts to move under gravity.

The continuity, momentum, and energy equations for the fluid are [An et al. (2008); Liu et al. (2009); Liu et al. (2010)]:

$$
\begin{aligned}
& \nabla \cdot v=0 \\
& \rho_{0}\left(\frac{\partial v}{\partial t}+v \cdot \nabla v\right)=-\nabla p+\mu \nabla^{2} v+\rho g \\
& \rho_{0} c_{p}\left(\frac{\partial T}{\partial t}+v \cdot \nabla T\right)=k \nabla^{2} T
\end{aligned}
$$




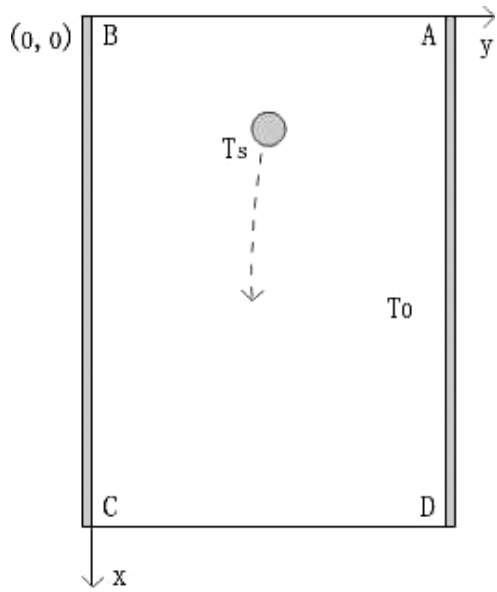

Fig. 1. Schematic of the computational domain. The vertical channel has a width of $8 d$ and is sufficiently long, so that top and bottom boundaries have little influence on the motion of the particle.

The fluid density $\rho$ varies with the temperature $T$ according to the Boussinesq approximation:

$$
\rho=\rho_{0}\left[1-\beta\left(T-T_{0}\right)\right]
$$

where $\rho_{0}$ is the fluid density at the far-field temperature $T_{0}$ and $\beta$ is the coefficient of thermal expansion for the fluid.

The particle translates and rotates according to the following equations:

$$
\begin{aligned}
m_{i} \frac{d V_{i}}{d t} & =G_{i}+F_{i}, \\
I_{i} \frac{d \Omega_{i}}{d t} & =T_{i},
\end{aligned}
$$

where $i$ denotes the $i$ th particle, $m_{i}$ and $I_{i}$ is the mass and moment of inertia of the particle, $V_{i}$ and $\Omega_{i}$ are its velocity and angular velocity, and $G_{i}, F_{i}$, and $T_{i}$ are the body force, hydrodynamic force, and moment, respectively.

On the four sides of the computational domain we impose the following conditions (see Fig. 1):

$$
\begin{gathered}
v=0, \quad T=T_{0} \text { on } \mathrm{BC}, \mathrm{CD}, \mathrm{DA}, \quad \text { and } \\
\tau_{x y}=\tau_{y y}=0, \quad \frac{\partial T}{\partial x}=0 \quad \text { on } \mathrm{AB},
\end{gathered}
$$

where $\tau$ represents total stress components. During the simulation, the computational domain is redefined at each time step according to the positions of the particles. The size of the computational domain and the particles positions to the up and bottom are fixed. Therefore, $v=0$ on CD implies that the channel is so deep that the falling particles drive no net flow rate. In our study, $m_{i}$ and $I_{i}$ equal to $\pi \rho_{s} d^{2} / 4$ and $\pi \rho_{s} d^{4} / 32$, respectively. 
The dimensionless parameters are:

Reynolds number:

$$
R e=\frac{\rho_{0} U d}{\mu},
$$

Grashof number:

$$
G r=\frac{\rho_{0}^{2} \beta \Delta T d^{3} g}{\mu^{2}},
$$

Prandtl number:

$$
\operatorname{Pr}=\frac{\mu c p}{k}, \quad \text { and }
$$

Rayleigh number:

$$
R a=G r P r .
$$

It is clear that the problem involves coupled physics with fluid flow, thermal convection, and mass transfer of moving particles. To effectively investigate such fluid-particle two-phase flow problem on micro-scale, we have developed a direct numerical simulation method, in which the full Navier-Stokes equations are solved using finite-element formulation for fluid flow. The Arbitrary Lagrangian-Eulerian (ALE) method is used in this paper to compute the fluid flow. The motion of particles is obtained by integrating their equations of motion using the hydrodynamic force and torque and the motion of particles also provides boundary condition for fluid flow. An unstructured mesh of triangular elements generated by the Delaunary-Voronoi method is used. As the particles move, the mesh moves and deforms according to a mesh velocity determined by a Laplace equation. A collision rule is introduced to account for solid contact due to surface roughness. The governing equations are discretized using a Galerkin formation. Residues from the solid and fluid momentum equations are combined into one weak form. In this way, the hydrodynamic force and moment cancel out between the two phases and need not be computed explicitly. The position of the particles is updated explicitly but their velocity is determined implicitly along with the fluid velocity to ensure stability of the scheme. The time step is automatically adjusted according to the velocity and acceleration of the particles. The nonlinearity in convection is handled by Newton iteration and the linear systems are solved using an iterative algorithm. The effectiveness of the numerical scheme has been validated using a series of examples with thermal convection [Liu et al. (2009)].

\section{Results and Discussions}

To investigate how thermal convection affects the hydrodynamic force on a particle and hence its motion, three cases are simulated including non-convection isothermal particle sedimentation $\left(T_{0}=T_{s}\right)$, cold particle sedimentation in hot $\left(T_{0}>T_{s}\right)$ fluid, and hot particle sedimentation in cold $\left(T_{0}<T_{s}\right)$ fluid, respectively. The elliptical particle with an initial major axis vertical to $x$-axis is marked "diaa $=2$, diab $=1$," 
and in contrast, the elliptical particle with an initial major axis parallel to $x$-axis is marked "diaa $=1$, diab $=2$." The solid-to-fluid destiny ratio $\rho_{s} / \rho_{0}=1.00464$.

\subsection{Non-convection particle sedimentation}

Figure 2 shows that the elliptical particle whose initial major axis is vertical to $x$-axis accelerates monotonically after release, then reaches maximal velocity and settles along the center of the channel at constant velocity. The elliptical particle whose major axis is parallel to $x$-axis accelerates to its maximum after release, then decreases to a constant value, migrates away from the centerline at the beginning

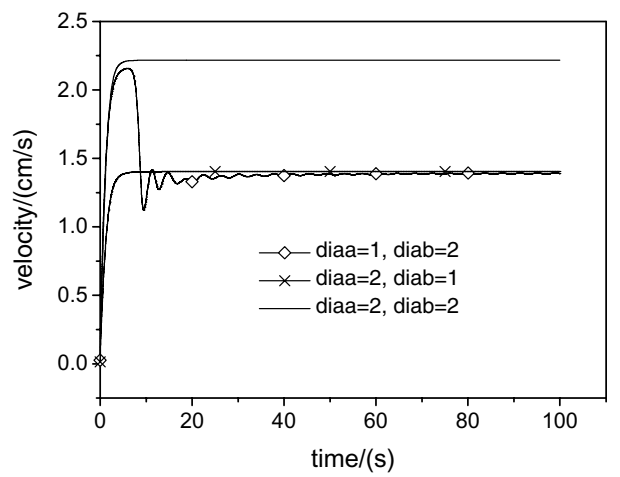

(a)

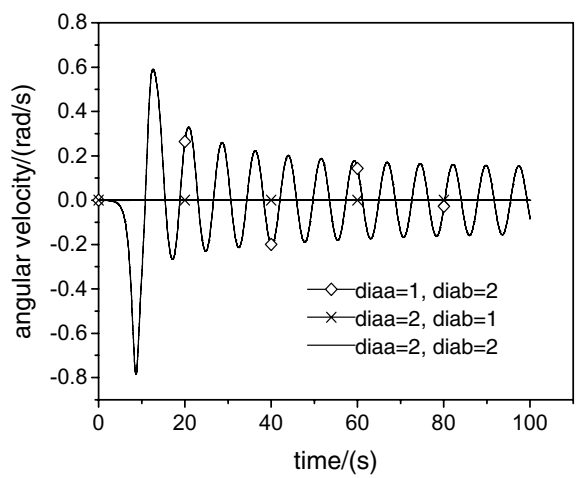

(c)

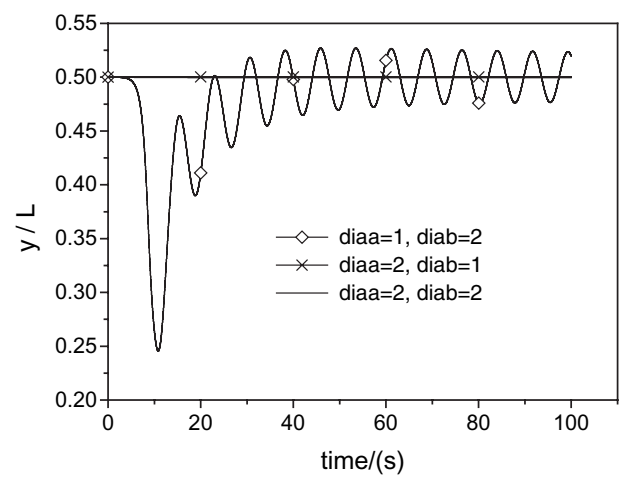

(b)

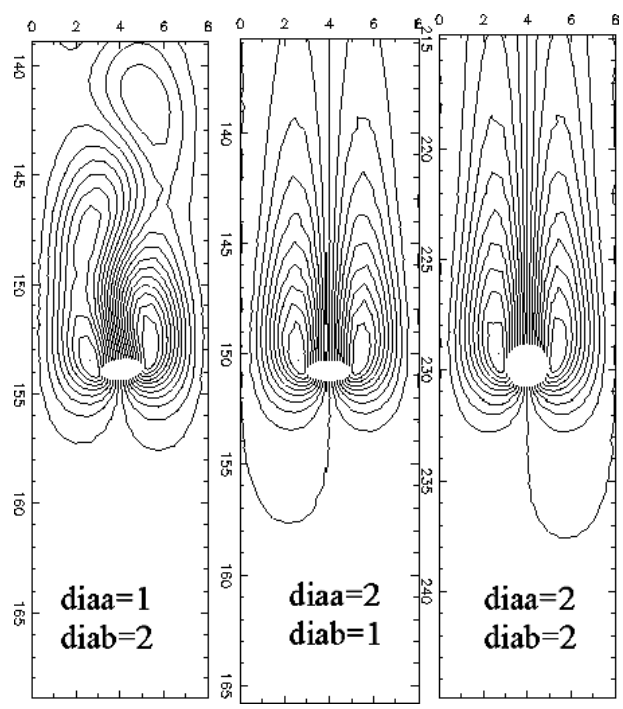

(d)

Fig. 2. Non-convection particle sedimentation: (a) settling trajectories, (b) variation of vertical velocities with time, (c) angular velocity component, and (d) flow field at $100 \mathrm{~s}$. 
accompanying with increasing angular velocity. The angular velocity attains maximum, as the particle nears the end of the migration and then the particle moves back to the centerline. The particle rotates from parallel to vertical to the $x$-axis during the process and displays weak and somewhat irregular lateral oscillations about the centerline. It finally rotates about the axis vertical to $x$-axis and the oscillation dies out gradually. The terminal velocities of the two elliptical particles are equal, being $1.403 \mathrm{~cm} / \mathrm{s}$, which is smaller than that of the circle particle $2.217 \mathrm{~cm} / \mathrm{s}$.

The particle oscillations are caused by the vortex shedding, the inertial lift, and torque associated with the orientation of the elliptical particle when changing the major axis location, which can be revealed by the flow field. The circular particle and the elliptical particle whose initial major axis vertical to $x$-axis accompany with symmetric and steady vortex shedding without rotation. The trail structure of the elliptical particle whose major axis is parallel to $x$-axis diversifies constantly. Besides, the particles will be confined by the wall during sedimentation, which forces the particles to equilibrate at the center of the channel.

\subsection{Cold particle sedimentation in hot $\left(T_{0}>T_{S}\right)$ fluid}

The elliptical particle whose initial major axis is vertical to $\mathrm{x}$-axis straight falls down along the centerline in hot fluid initially, then develops a regular lateral oscillation (Fig. 3(a)). The amplitudes of oscillation increase from zero to $0.2 d$ for the circular particle and $0.7 d$ for the elliptical particle. The angular velocity is not similar to that observed in the above case with isothermal particles. The particles rotate about the equilibrium position vertical to $x$-axis (Fig. $3(\mathrm{a})$ ). The elliptical particle whose major axis is parallel to $x$-axis migrates to the wall and then achieves regular oscillation about the centerline. For both cases of elliptical particles, the final amplitudes of oscillation are the same.

The regular particle oscillation is caused by the combination of downward thermal convection and the upward external flow in the boundary layer. The flow in

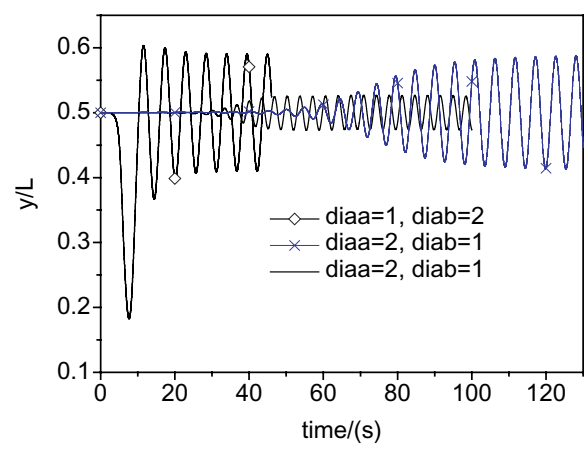

(a)

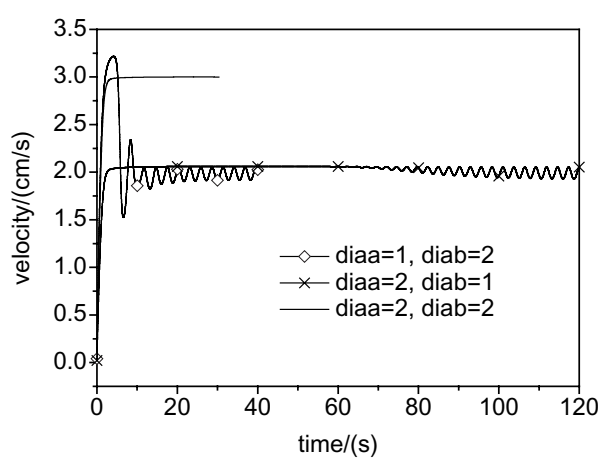

(b)

Fig. 3. Cold particle sedimentation in hot $\left(T_{0}>T_{s}\right)$ fluid: (a) settling trajectories, (b) variation of vertical velocities upon time, (c) angular velocity component, and (d) flow field and temperature contour at $100 \mathrm{~s}$. 


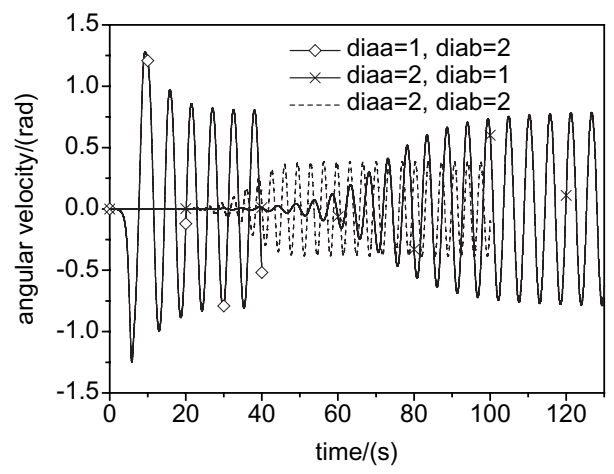

(c)

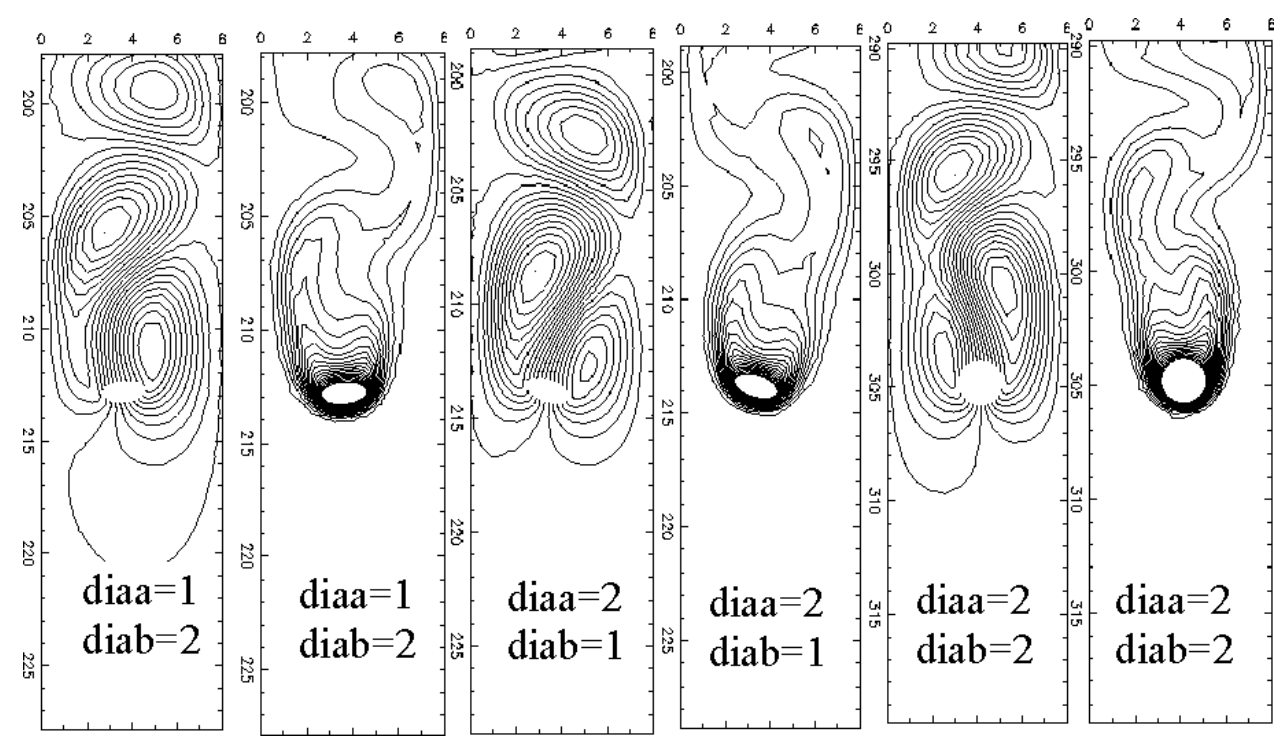

(d)

Fig. 3. (Continued)

the rear and front of the particle brings reverse forces, which generates "twist equilibrium." If there is lateral disturbance to the right during the sedimentation, the downward and upward flow will shift to the left of the particle, which results in an asymmetric force on the particle's left which pushes the particle to the right. When the particle approaches the solid wall, the repulsive force on the particle is stronger. Sometimes, the resultant forces point to right, which push the particle to the centerline. These form the regular oscillation periodically. The major axis is vertical to $x$-axis during sedimentation when the particle achieves equilibrium. The lateral oscillation and angular velocity are the same in both cases with elliptical particles. 
The cold particles have greater vertical velocities, lateral oscillation, and angular velocity in contrast to corresponding isothermal particles, the reason is that downward thermal convection speeds up the sedimentation. The vortex shedding also contributes to the movement style of the particles (Fig. 3(d)).

\subsection{Hot particle sedimentation in cold $\left(T_{0}<T_{S}\right)$ fluid}

The circular particle settles along the centerline after release; its velocity is smaller than that of the corresponding isothermal particle, increases with time, and then reaches the maximum value. There is no angular velocity all the time. The elliptical particles move away from centerline and then develop a regular lateral oscillation about an off-center equilibrium position (Fig. 4). The particle whose major axis is parallel to $x$-axis achieves equilibrium first. The vertical velocities and angular velocities develop a regular oscillation too. During the sedimentation of hot particle

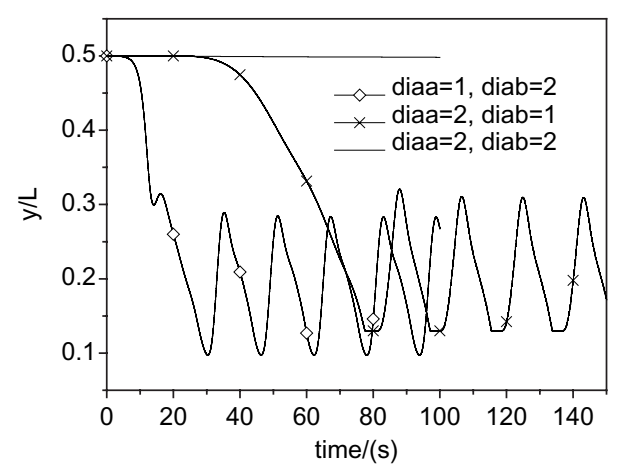

(a)

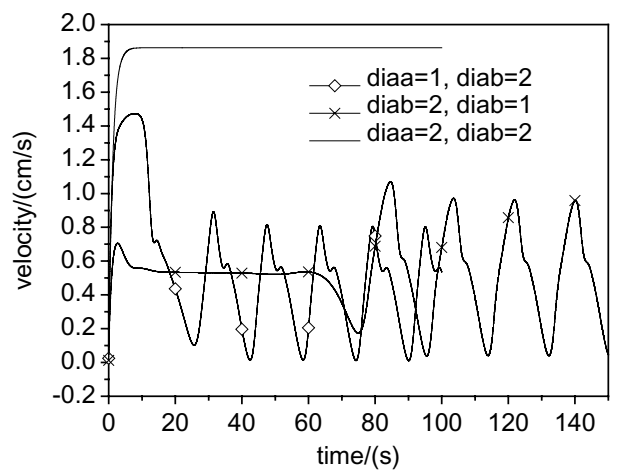

(b)

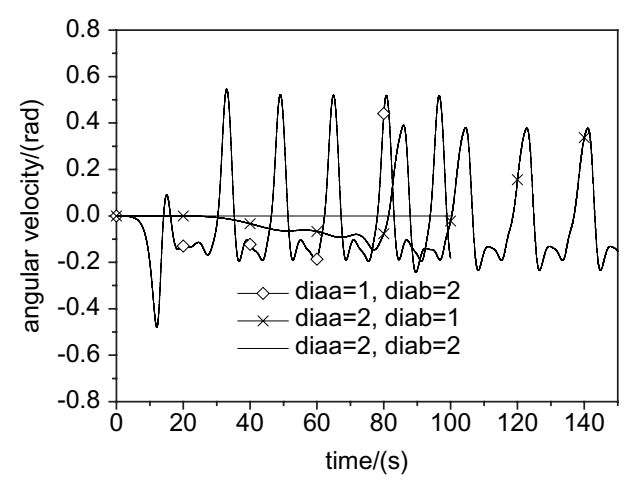

(c)

Fig. 4. Hot particle sedimentation in cold $\left(T_{0}<T_{s}\right)$ fluid: (a) settling trajectories, (b) variation of vertical velocities upon time, (c) angular velocity component, and (d) flow field and temperature contour at $100 \mathrm{~s}$. 

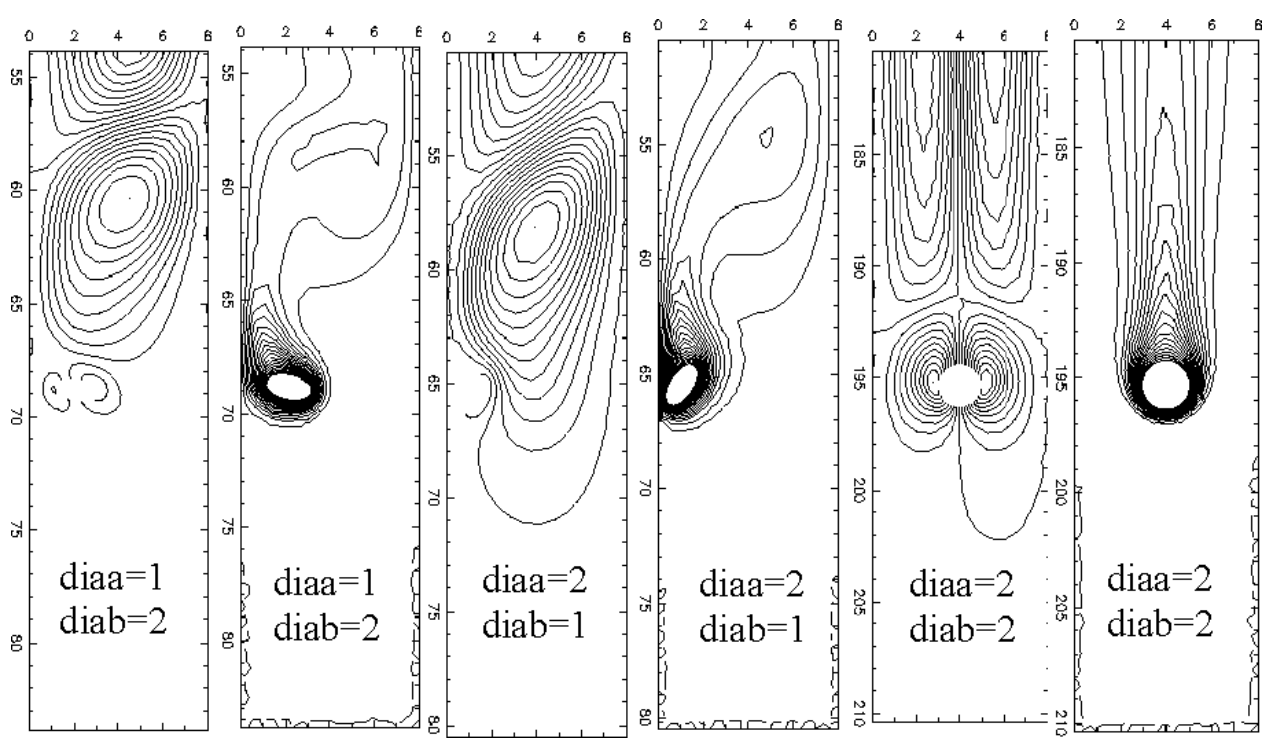

(d)

Fig. 4. (Continued)

in the cold fluid, the warm wake forms a strong upward thermal plume; the hot layer of fluid next to the particles carry upward momentum; therefore, the hot particles have smaller vertical velocities and lateral and angular velocities are in contrast to isothermal particles. The off-center equilibrium position sedimentation is resulted from the warm wake: if the jet is displaced to the left, say, the faster flow near the left wall will reduce the local pressure, the lateral migration is driven by the high pressure on the right side as well as the strong shear stress on the upper right of the particle. Moreover, when the particle approaches the solid wall, the repulsive force on the particle is stronger. Then, at certain lateral location, the particle achieves off-center equilibrium. The circular particle has a bigger velocity and the thermal convection effect is suppressed; therefore, it settles steadily without oscillation.

\section{Conclusions}

In this paper, the sedimentation processes of elliptical particles in a Newtonian fluid were directly simulated in a vertical channel at three different scenarios with isotherm, hot, and cold Newtonian fluids. The flow behavior is affected by vortex shedding, inertial lift, wall confinement, and torque associated with the orientation of the particle for elliptical particles when changing the major axis location. It is shown that elliptical particles can lead to more complicated flow behavior than circular particles. For isothermal particle sedimentation, the final direction of major axis of the elliptical particle is vertical to $x$-axis. For cold particle sedimentation in hot fluid, the elliptical particle eventually develops a regular lateral oscillation along 
the centerline of the channel with the same oscillation amplitudes for both particle orientations. The downward thermal convection speeds up the sedimentation process; therefore, the cold particles have greater vertical velocities, lateral oscillation, and angular velocity in contrast to isothermal particles. For hot particle sedimentation in cold fluid, the elliptical particles develop a regular lateral oscillation about an off-center equilibrium position after release and the vertical and angular velocities change periodically. The warm wake forms a strong upward thermal plume that is prominent at low Reynolds number. The hot particles have smaller vertical velocities and lateral and angular velocities are in contrast to the isothermal particles. Besides, similar results were obtained while the ratio of $a / b$ is slightly larger than two, and further studies include applying this method to moving fluids and/or non-Newtonian fluids.

\section{Acknowledgments}

This work has been supported by the National Natural Science Foundation of China (Grant No. 50976108, 10942004 and 11172306). And the Natural Science Foundation for Young Scientists of Shanxi Province, China (Grant No. 2008041002).

\section{References}

Acrivos, A. and Taylor, T. D. [1962] Heat and mass transfer from single spheres in Stokes flow, Phys. Fluid. 5, 387-394.

An, K., Chang, J. Z. and Liu, H. T. [2008] Direct numerical simulation of the influence by channel width in the sedimentation of a single particle, Port Waterway Eng. 418(8), 6-9.

Anthony, W. [2009] A DEM-DLM/FD method for direct numerical simulation of particulate flows: Sedimentation of polygonal isometric particles in a Newtonian fluid with collisions, Comput. Fluid. 38(8), 1608-1628.

Codina, R. and Houzeaux, G. [2009] The fixed-mesh ALE approach for the numerical approximation of flows in moving domains, J. Comp. Phys. 228, 1591-1611.

Deen, N. G. and Annaland, M. V. S. [2007] Review of discrete particle modeling of fluidized beds, Chem. Sci. 62, 28-44.

Feng, F., Hu, H. H. and Joseph, D. D. [1994] Direct simulation of initial value problems for the motion of solid bodies in a Newtonian fluid. Part 1. Sedimentation, J. Fluid. Mech. 261, 95-134.

Gan, H., Chang, J. Z., Feng, J. J. and Howard, H. H. [2003] Direct numerical simulation of the sedimentation of solid particles with thermal convection, J. Fluid. Mech. 481, $385-411$.

Jae, M. K. and Ronald, J. P. [2004] Dissipative particle dynamics simulation of flow around spheres and cylinders at finite Reynolds numbers, Chem. Eng. Sci. 59, 4155-4168.

Jog, M. A., Ayyaswamy, P. S. and Cohen, I. M. [1996] Evaporation and combustion of a slowly moving liquid fuel droplet: High-order theory, J. Fluid Mech. 307, 135-165.

Kuehn, T. H. and Goldstein, R. J. [1976] An experimental and theoretical study of natural convection in the annulus between horizontal concentric cylinder, J. Fluid Mech. 74(4), $695-719$.

Lin, J. Z., Lin, J. and Shi, X. [2002] Research on the effect of cylinder particles on the turbulent properties in particulate flows, Appl. Math. Mech. 23(5), 542-548. 
Liu, M. Y., Tang, X. P. and Jiang, P. [2004] Studies on the hydrodynamic and heat transfer in a vapor-liquid-solid flow boiling system with a CCD measuring technique, Chem. Eng. Sci. 59, 889-899.

Liu, H. T., Tong, Z. H., An, K. and Ma, L. Q. [2009] Direct simulation of the influence on the motion of particles in a Newtonian fluid by melting and convection, Acta Phys. Sin. 58(9), 6369-6375.

Liu, H. T., Chang, J. Z., An, K. and Su, T. X. [2010] Direct numerical simulation of the sedimentation of two particle with thermal convection, Acta Phys. Sin. 59(3), 18771883.

Mcleod, P., Riley, D. S. and Sparks, R. S. J. [1996] Melting of a sphere in hot fluid, J. Fluid Mech. 327, 393-409.

Mezhericher, M., Levy, A. and Borde, I. [2008] Heat and mass transfer of single droplet/wet particle drying, Chem. Eng. Sci. 63, 12-23.

Shigeo, A. and Hideo, I. [2001] Melting characteristics of ice water slurry by warm air bubbling, Int. J. Therm. Sci. 40, 724-737.

Takeuchi, S., Morita, I. and Kajishima, T. [2008] Motion of particle agglomerate involving interparticle force in diluter suspension, Power Tech. 184, 232-240.

You, C. F., Qi, H. Y. and Xu, X. C. [2003] Numerical simulation of drag force on nonspherical particle in gas-particle two-phase flow, J. Chem. Indust. Eng. 54(2), 188-191. 